TRANSACTIONS OF THE

AMERICAN MATHEMATICAL SOCIETY

Volume 362, Number 6, June 2010, Pages 2859-2873

S 0002-9947(10)04744-6

Article electronically published on January 20, 2010

\title{
LARGE, GLOBAL SOLUTIONS TO THE NAVIER-STOKES EQUATIONS, SLOWLY VARYING IN ONE DIRECTION
}

\author{
JEAN-YVES CHEMIN AND ISABELLE GALLAGHER
}

\begin{abstract}
In two earlier papers by the authors, classes of initial data for the three dimensional, incompressible Navier-Stokes equations were presented, generating a global smooth solution although the norm of the initial data may be chosen arbitrarily large. The aim of this article is to provide new examples of arbitrarily large initial data giving rise to global solutions, in the whole space. Contrary to the previous examples, the initial data has no particular oscillatory properties, but varies slowly in one direction. The proof uses the special structure of the nonlinear term of the equation.
\end{abstract}

\section{INTRODUCTION}

The purpose of this paper is to use the special structure of the tridimensional Navier-Stokes equations to prove the global existence of smooth solutions for a class of (large) initial data which are slowly varying in one direction. Before entering into further detail, let us recall briefly some classical facts on the global wellposedness of the incompressible Navier-Stokes equations in the whole space $\mathbb{R}^{3}$. The equations are

$$
(N S)\left\{\begin{array}{c}
\partial_{t} u+u \cdot \nabla u-\Delta u=-\nabla p \\
\operatorname{div} u=0 \\
u_{\mid t=0}=u_{0}
\end{array}\right.
$$

where $u=\left(u^{1}, u^{2}, u^{3}\right)=\left(u^{h}, u^{3}\right)$ is a time dependent vector field on $\mathbb{R}^{3}$. The divergence free condition determines $p$ through the relation

$$
-\Delta p=\sum_{1 \leq j, k \leq 3} \partial_{j} \partial_{k}\left(u^{j} u^{k}\right) .
$$

This relation allows us to put the system $(N S)$ in the more general form

$$
(G N S)\left\{\begin{aligned}
\partial_{t} u-\Delta u & =Q(u, u) \\
u_{\mid t=0} & =u_{0}
\end{aligned}\right.
$$

where $Q(v, w) \stackrel{\text { def }}{=} \sum_{1 \leq j, k \leq 3} Q_{j, k}(D)\left(v^{j} w^{k}\right)$ and $Q_{j, k}(D)$ are smooth homogeneous Fourier multipliers of order 1.

Moreover, this system has the following scaling invariance: if $(u, p)$ is a solution on the time interval $[0, T)$, then $\left(u_{\lambda}, p_{\lambda}\right)$ defined by

$$
u_{\lambda}(t, x) \stackrel{\text { def }}{=} \lambda u\left(\lambda^{2} t, \lambda x\right) \quad \text { and } \quad p_{\lambda}(t, x) \stackrel{\text { def }}{=} \lambda^{2} p\left(\lambda^{2} t, \lambda x\right)
$$

Received by the editors October 29, 2007.

2000 Mathematics Subject Classification. Primary 35Q30, 76D05, 76D03.

Key words and phrases. Navier-Stokes equations, global wellposedness. 
is a solution on the time interval $\left[0, \lambda^{-2} T\right)$. Of course, any smallness condition on the initial data that ensures global solutions must be invariant under the above scaling transformation. The search for the "best" smallness condition is a long story that was initiated in the seminal paper of J. Leray (see [14]) and continued in particular by H. Fujita and T. Kato in [5], Y. Giga and T. Miyakawa in [8], and M. Cannone, Y. Meyer and F. Planchon in [1]. This led to the following theorem proved by H. Koch and D. Tataru in [11. In the statement of the theorem, $P(x, R)$ stands for the parabolic set $\left[0, R^{2}\right] \times B(x, R)$ where $B(x, R)$ is the ball centered at $x$ of radius $R$.

Theorem 1 ([1] $)$. If the initial data $u_{0}$ is such that

$$
\left\|u_{0}\right\|_{B M O^{-1}}^{2} \stackrel{\text { def }}{=} \sup _{t>0} t\left\|e^{t \Delta} u_{0}\right\|_{L^{\infty}}^{2}+\sup _{\substack{x \in \mathbb{R}^{3} \\ R>0}} \frac{1}{R^{3}} \int_{P(x, R)}\left|\left(e^{t \Delta} u_{0}\right)(t, y)\right|^{2} d y
$$

is small enough, then there exists a global smooth solution to (GNS).

A typical example of application of this theorem is the initial data

$$
u_{0}^{\varepsilon}(x) \stackrel{\text { def }}{=} \cos \left(\frac{x_{3}}{\varepsilon}\right)\left(\partial_{2} \phi\left(x_{1}, x_{2}\right),-\partial_{1} \phi\left(x_{1}, x_{2}\right), 0\right) \quad \text { with } \quad \phi \in \mathcal{S}\left(\mathbb{R}^{2}\right),
$$

provided $\varepsilon$ is small enough (see for example 44 for a proof). The above theorem is probably the end point for the following reason, as observed for instance in 4. If $B$ is a Banach space continuously included in the space $\mathcal{S}^{\prime}$ of tempered distributions on $\mathbb{R}^{3}$ such that for any $(\lambda, a) \in \mathbb{R}_{\star}^{+} \times \mathbb{R}^{3}\|f(\lambda(\cdot-a))\|_{B}=\lambda^{-1}\|f\|_{B}$, then $\|\cdot\|_{B} \leq C \sup t^{\frac{1}{2}}\left\|e^{t \Delta} u_{0}\right\|_{L^{\infty}}$. The second term in the definition of the $B M O^{-1}$ norm given in (1.1) merely expresses the fact that the first Picard iterate should be locally square integrable in space and time.

These results on global existence under a smallness condition do not use the special structure of the incompressible Navier-Stokes system and are valid for the larger class of systems of type $(G N S)$. The purpose of this paper is to provide a class of examples of large initial data which give rise to global smooth solutions for the system $(N S)$ itself, not for the larger class $(G N S)$. In what follows, an initial data $u_{0}$ will be considered "large" if

$$
\left\|u_{0}\right\|_{\dot{B}_{\infty, \infty}^{-1}} \stackrel{\text { def }}{=} \sup _{t>0} t^{\frac{1}{2}}\left\|e^{t \Delta} u_{0}\right\|_{L^{\infty}}
$$

is not small.

Such initial data, in the spirit of the example provided by (1.2), are exhibited in [4] (see also [3] for the periodic case). In particular, the following theorem is proved in 4 .

Theorem $2(4])$. Let $\phi \in \mathcal{S}\left(\mathbb{R}^{3}\right)$ be a given function, and consider two real numbers $\varepsilon$ and $\alpha$ in $] 0,1[$. Define

$$
\varphi^{\varepsilon}(x)=\frac{(-\log \varepsilon)^{\frac{1}{5}}}{\varepsilon^{1-\alpha}} \cos \left(\frac{x_{3}}{\varepsilon}\right) \phi\left(x_{1}, \frac{x_{2}}{\varepsilon^{\alpha}}, x_{3}\right) .
$$

Then for $\varepsilon$ small enough, the smooth, divergence free vector field

$$
u_{0}^{\varepsilon}(x)=\left(\partial_{2} \varphi^{\varepsilon}(x),-\partial_{1} \varphi^{\varepsilon}(x), 0\right)
$$

satisfies $\lim _{\varepsilon \rightarrow 0}\left\|u_{0}^{\varepsilon}\right\|_{\dot{B}_{\infty, \infty}^{-1}}=\infty$ and generates a unique global solution to $(N S)$. 
It is important to notice that the proof uses in a crucial way the algebraic structure of the nonlinear term $u \cdot \nabla u$, but uses neither the energy estimate nor the fact that the two dimensional, incompressible Navier-Stokes is globally wellposed. We refer to [15], 10] and [17] for other examples of large initial data generating global solutions: in the first case the initial data is chosen so as to transform the equation into a rotating fluid equation, whereas in the other two studies the equations are posed in a thin domain. In all cases, as well as in the present paper, the global wellposedness of the two dimensional equation is an important ingredient in the proof. In [6] and 9], the case of initial data close to a bidimensional vector field is studied in the periodic case. References [16] and [7] also present global stability results for arbitrarily large initial data generating a global solution (with some decay in time in the case of [16; note that Theorem 4 in [16] has the same flavour as Theorem 3 stated below in the sense that the initial data is a perturbation of a $2 \mathrm{D}$ vector field, depending only on the horizontal variable). Finally we also recall well-known examples where a specific geometrical assumption allows global wellposedness to be proved, such as in [12, [13, or [18.

The class of examples we exhibit here is of the following type: there is no particular geometric assumption (the equations are set in $\mathbb{R}^{3}$ ), but the data are close to a two dimensional flow in the sense that they are slowly varying in one direction (the vertical one). More precisely, the aim of this paper is to prove the following theorem.

Theorem 3. Let $v_{0}^{h}=\left(v_{0}^{1}, v_{0}^{2}\right)$ be a horizontal, smooth divergence free vector field on $\mathbb{R}^{3}$ (i.e. $v_{0}^{h}$ is in $L^{2}\left(\mathbb{R}^{3}\right)$, as well as all its derivatives) such that it and all its derivatives belong to $L^{2}\left(\mathbb{R}_{x_{3}} ; \dot{H}^{-1}\left(\mathbb{R}^{2}\right)\right)$; let $w_{0}$ be a smooth divergence free vector field on $\mathbb{R}^{3}$. Then there exists a positive $\varepsilon_{0}$ such that if $\varepsilon \leq \varepsilon_{0}$, the initial data

$$
u_{0}^{\varepsilon}(x)=\left(v_{0}^{h}+\varepsilon w_{0}^{h}, w_{0}^{3}\right)\left(x_{h}, \varepsilon x_{3}\right)
$$

generates a unique, global solution $u^{\varepsilon}$ of $(N S)$.

\section{Remarks}

- A typical example of a vector field $v_{0}^{h}$ satisfying the hypothesis is $v_{0}^{h}=$ $\left(-\partial_{2} \phi, \partial_{1} \phi\right)$ where $\phi$ is a function of the Schwarz class $\mathcal{S}\left(\mathbb{R}^{3}\right)$.

- This class of examples of initial data corresponds to a "well prepared" case. The "ill prepared" case would correspond to where the horizontal divergence of the initial data is of size $\varepsilon^{\alpha}$ with $\alpha$ less than 1 , and the vertical component of the initial data is of size $\varepsilon^{\alpha-1}$. This case is certainly very interesting to understand, but it probably goes far beyond the methods used in this paper.

- As in 3, one may wonder what influence a large Reynolds number would have on the initial data. Actually since the 2D Navier-Stokes equation is wellposed regardless of the Reynolds number, the initial profiles $v_{0}^{h}$ and $w_{0}$ can be chosen arbitrarily large with respect to the Reynolds number. In this case, $\varepsilon_{0}$ in Theorem 3 will depend on the Reynolds number.

- We have to check that the initial data may be large. This is ensured by the following proposition.

Proposition 1.1. Let $(f, g)$ be in $\mathcal{S}\left(\mathbb{R}^{2}\right) \times \mathcal{S}(\mathbb{R})$. Let us define $h^{\varepsilon}\left(x_{h}, x_{3}\right) \stackrel{\text { def }}{=}$ $f\left(x_{h}\right) g\left(\varepsilon x_{3}\right)$. We have, if $\varepsilon$ is small enough,

$$
\left\|h^{\varepsilon}\right\|_{\dot{B}_{\infty, \infty}^{-1}\left(\mathbb{R}^{3}\right)} \geq \frac{1}{4}\|f\|_{\dot{B}_{\infty, \infty}^{-1}\left(\mathbb{R}^{2}\right)}\|g\|_{L^{\infty}(\mathbb{R})} .
$$


Proof. By the definition of $\|\cdot\|_{\dot{B}_{\infty}^{-1}, \infty}\left(\mathbb{R}^{3}\right)$ given by (1.3), we have to bound from below the quantity $\left\|e^{t \Delta} h^{\varepsilon}\right\|_{L^{\infty}\left(\mathbb{R}^{3}\right)}$. Let us write

$$
\left(e^{t \Delta} h^{\varepsilon}\right)(t, x)=\left(e^{t \Delta_{h}} f\right)\left(t, x_{h}\right)\left(e^{t \partial_{3}^{2}} g\right)\left(\varepsilon^{2} t, \varepsilon x_{3}\right) .
$$

Let us consider a positive time $t_{0}$ such that

$$
t_{0}^{\frac{1}{2}}\left\|e^{t_{0} \Delta_{h}} f\right\|_{L^{\infty}\left(\mathbb{R}^{2}\right)} \geq \frac{1}{2}\|f\|_{\dot{B}_{\infty, \infty}^{-1}\left(\mathbb{R}^{2}\right)} .
$$

Then we have

$$
\begin{aligned}
t_{0}^{\frac{1}{2}}\left\|e^{t_{0} \Delta} h^{\varepsilon}\right\|_{L^{\infty}\left(\mathbb{R}^{3}\right)} & =t_{0}^{\frac{1}{2}}\left\|e^{t_{0} \Delta_{h}} f\right\|_{L^{\infty}\left(\mathbb{R}^{2}\right)}\left\|\left(e^{t_{0} \partial_{3}^{2}} g\right)\left(\varepsilon^{2} t_{0}, \varepsilon \cdot\right)\right\|_{L^{\infty}(\mathbb{R})} \\
& \geq \frac{1}{2}\|f\|_{\dot{B}_{\infty, \infty}^{-1}\left(\mathbb{R}^{2}\right)}\left\|e^{\varepsilon^{2} t_{0} \partial_{3}^{2}} g\right\|_{L^{\infty}(\mathbb{R})} .
\end{aligned}
$$

As $\lim _{\varepsilon \rightarrow 0} e^{\varepsilon^{2} t_{0} \partial_{3}^{2}} g=g$ in $L^{\infty}(\mathbb{R})$, the proposition is proved.

Structure of the paper: The proof of Theorem 3 is achieved in the next section, assuming two crucial lemmas. The proof of these lemmas is postponed to Sections 3 and 4 respectively.

Notation: If $A$ and $B$ are two real numbers, we shall write $A \lesssim B$ if there is a universal constant $C$, which does not depend on varying parameters of the problem, such that $A \leq C B$. If $A \lesssim B$ and $B \lesssim A$, then we shall write $A \sim B$.

If $v_{0}$ is a vector field, then we shall denote by $C_{v_{0}}$ a constant depending only on the norm of $v_{0}$. Similarly we shall use the notation $C_{v_{0}, w_{0}}$ if the constant depends on the norms of two vector fields $v_{0}$ and $w_{0}$, etc.

A function space with a subscript " $h$ " (for "horizontal") will denote a space defined on $\mathbb{R}^{2}$, while the subscript "v" (for "vertical") will denote a space defined on $\mathbb{R}$. For instance, $L_{h}^{p} \stackrel{\text { def }}{=} L^{p}\left(\mathbb{R}^{2}\right), L_{v}^{q} \stackrel{\text { def }}{=} L^{q}(\mathbb{R})$, and similarly for Sobolev spaces and mixed spaces such as $L_{v}^{p} L_{h}^{q}$ or $L_{v}^{p} \dot{H}_{h}^{\sigma}$.

\section{Proof of THE THEOREM}

The proof of Theorem 3 consists of constructing an approximate solution to $(N S)$ as a perturbation to the 2D Navier-Stokes system. Following the idea that we are close to the two dimensional, periodic incompressible Navier-Stokes system, let us define $\left(\underline{v}^{h}, \underline{p}_{0}\right)$ as the solution of the following system, where $y_{3} \in \mathbb{R}$ is a parameter:

$$
\left(N S 2 D_{3}\right)\left\{\begin{array}{c}
\partial_{t} \underline{v}^{h}+\underline{v}^{h} \cdot \nabla_{h} \underline{v}^{h}-\Delta_{h} \underline{v}^{h}=-\nabla_{h} \underline{p}_{0} \\
\operatorname{div}_{h} \underline{v}^{h}=0 \\
\underline{v}_{\mid t=0}^{h}=v_{0}^{h}\left(\cdot, y_{3}\right) .
\end{array}\right.
$$

This system is globally wellposed for any $y_{3} \in \mathbb{R}$, and the solution is smooth in (two dimensional) space and in time. Let us consider the solution $\left(\underline{w}^{\varepsilon}, \underline{p}_{1}\right)$ of the linear equation

$$
\left(T_{\underline{v}}^{\varepsilon}\right)\left\{\begin{array}{c}
\partial_{t} \underline{w}^{\varepsilon}+\underline{v}^{h} \cdot \nabla_{h} \underline{w}^{\varepsilon}-\Delta_{h} \underline{w}^{\varepsilon}-\varepsilon^{2} \partial_{3}^{2} \underline{w}^{\varepsilon}=-\left(\nabla^{h} \underline{p}_{1}, \varepsilon^{2} \partial_{3} \underline{p}_{1}\right) \quad \text { in } \mathbb{R}^{+} \times \mathbb{R}^{3} \\
\operatorname{div} \underline{w}^{\varepsilon}=0 \\
\underline{w}_{\mid t=0}^{\varepsilon}=w_{0},
\end{array}\right.
$$

and define the approximate solution

$$
\begin{aligned}
& v_{a p p}^{\varepsilon}(t, x)=\left(\left(\underline{v}^{h}, 0\right)+\varepsilon\left(\underline{w}^{\varepsilon, h}, \varepsilon^{-1} \underline{w}^{\varepsilon, 3}\right)\right)\left(t, x_{h}, \varepsilon x_{3}\right) \quad \text { and } \\
& p_{\text {app }}^{\varepsilon}(t, x)=\left(\underline{p}_{0}+\varepsilon \underline{p}_{1}\right)\left(t, x_{h}, \varepsilon x_{3}\right) .
\end{aligned}
$$


Finally let us consider the unique smooth solution $u^{\varepsilon}$ of $(N S)$ associated with the initial data $u_{0}^{\varepsilon}$ on its maximal time interval of existence $\left[0, T_{\varepsilon}\right)$. The proof of Theorem 3 consists of proving global-in-time estimates on $v_{a p p}^{\varepsilon}$, in order to prove that $R^{\varepsilon} \stackrel{\text { def }}{=} u^{\varepsilon}-v_{a p p}^{\varepsilon}$ remains small globally in time; this ensures the global regularity for $(N S)$.

More precisely, the proof of Theorem 3 relies on the following two lemmas, whose proofs are postponed to Sections 3 and 4 respectively.

Lemma 2.1. The vector field $v_{a p p}^{\varepsilon}$ defined in (2.1) satisfies the following estimate:

$$
\left\|v_{a p p}^{\varepsilon}\right\|_{L^{2}\left(\mathbb{R}^{+} ; L^{\infty}\left(\mathbb{R}^{3}\right)\right)}+\left\|\nabla v_{a p p}^{\varepsilon}\right\|_{L^{2}\left(\mathbb{R}^{+} ; L_{v}^{\infty} L_{h}^{2}\right)} \leq C_{v_{0}, w_{0}} .
$$

Lemma 2.2. The vector field $R^{\varepsilon} \stackrel{\text { def }}{=} u^{\varepsilon}-v_{a p p}^{\varepsilon}$ satisfies the equation

$$
\left(E^{\varepsilon}\right)\left\{\begin{array}{c}
\partial_{t} R^{\varepsilon}+R^{\varepsilon} \cdot \nabla R^{\varepsilon}-\Delta R^{\varepsilon}+v_{a p p}^{\varepsilon} \cdot \nabla R^{\varepsilon}+R^{\varepsilon} \cdot \nabla v_{a p p}^{\varepsilon}=F^{\varepsilon}-\nabla q^{\varepsilon} \\
\operatorname{div} R^{\varepsilon}=0 \\
R_{\mid t=0}^{\varepsilon}=0
\end{array}\right.
$$

with $\left\|F^{\varepsilon}\right\|_{L^{2}\left(\mathbb{R}^{+} ; \dot{H}^{-\frac{1}{2}}\left(\mathbb{R}^{3}\right)\right)} \leq C_{v_{0}, w_{0}} \varepsilon^{\frac{1}{3}}$.

Let us postpone the proof of these lemmas and conclude the proof of Theorem 3 , We write, for any positive $\lambda$,

$$
\begin{aligned}
V_{\varepsilon}(t) & \stackrel{\text { def }}{=}\left\|v_{a p p}^{\varepsilon}(t, \cdot)\right\|_{L^{\infty}\left(\mathbb{R}^{3}\right)}^{2}+\left\|\nabla v_{a p p}^{\varepsilon}(t, \cdot)\right\|_{L_{v}^{\infty} L_{h}^{2}}^{2} \\
& \text { and } R_{\lambda}^{\varepsilon}(t) \stackrel{\text { def }}{=} \exp \left(-\lambda \int_{0}^{t} V_{\varepsilon}\left(t^{\prime}\right) d t^{\prime}\right) R^{\varepsilon}(t) .
\end{aligned}
$$

Lemma 2.1 implies that $I_{0} \stackrel{\text { def }}{=} \int_{0}^{\infty} V_{\varepsilon}(t) d t$ is finite. By an $\dot{H}^{\frac{1}{2}}$ energy estimate in $\mathbb{R}^{3}$, we get

$$
\begin{gathered}
\frac{1}{2} \frac{d}{d t}\left\|R_{\lambda}^{\varepsilon}(t)\right\|_{\dot{H}^{\frac{1}{2}}}^{2}+\left\|\nabla R_{\lambda}^{\varepsilon}(t)\right\|_{\dot{H}^{\frac{1}{2}}}^{2} \\
\leq-2 \lambda V_{\varepsilon}(t)\left\|R_{\lambda}^{\varepsilon}(t)\right\|_{\dot{H}^{\frac{1}{2}}}^{2}+e^{\lambda I_{0}}\left|\left(R_{\lambda}^{\varepsilon}(t) \cdot \nabla R_{\lambda}^{\varepsilon}(t) \mid R_{\lambda}^{\varepsilon}(t)\right)_{\dot{H}^{\frac{1}{2}}}\right| \\
+\left|\left(R_{\lambda}^{\varepsilon}(t) \cdot \nabla v_{a p p}^{\varepsilon}(t) \mid R_{\lambda}^{\varepsilon}(t)\right)_{\dot{H}^{\frac{1}{2}}}\right|+\left|\left(v_{a p p}^{\varepsilon}(t) \cdot R_{\lambda}^{\varepsilon}(t) \mid R_{\lambda}^{\varepsilon}(t)\right)_{\dot{H}^{\frac{1}{2}}}\right|+\left|\left(F^{\varepsilon}(t) \mid R_{\lambda}^{\varepsilon}(t)\right)_{\dot{H}^{\frac{1}{2}}}\right| .
\end{gathered}
$$

The estimate (i) in Lemma 1.1 of [2] claims in particular that for any $s \in]-d / 2, d / 2[$, any divergence free vector field $a$ in $d$ space dimensions and any function $b$, we have

$$
(a \cdot \nabla b \mid b)_{\dot{H}^{s}} \leq C\|\nabla a\|_{\dot{H}^{\frac{d}{2}-1}}\|b\|_{\dot{H}^{s}}\|\nabla b\|_{\dot{H}^{s}} .
$$

With $d=3$ and $s=1 / 2$, this implies that

$$
\left|\left(R_{\lambda}^{\varepsilon}(t) \cdot \nabla R_{\lambda}^{\varepsilon}(t) \mid R_{\lambda}^{\varepsilon}(t)\right)_{\dot{H}^{\frac{1}{2}}}\right| \lesssim\left\|R_{\lambda}^{\varepsilon}(t)\right\|_{\dot{H}^{\frac{1}{2}}}\left\|\nabla R_{\lambda}^{\varepsilon}(t)\right\|_{\dot{H}^{\frac{1}{2}}}^{2} .
$$

In order to estimate the other nonlinear terms, let us establish the following lemma.

Lemma 2.3. Let $a$ and $b$ be two vector fields. We have

$$
\left|(a \cdot \nabla b \mid b)_{\dot{H}^{\frac{1}{2}}}\right|+\left|(b \cdot \nabla a \mid b)_{\dot{H}^{\frac{1}{2}}}\right| \lesssim\left(\|a\|_{L^{\infty}}+\|\nabla a\|_{L_{v}^{\infty}\left(L_{h}^{2}\right)}\right)\|b\|_{\dot{H}^{\frac{1}{2}}}\|\nabla b\|_{\dot{H}^{\frac{1}{2}}} \cdot
$$


Proof. By definition of the $\dot{H}^{\frac{1}{2}}$ scalar product, we have

$$
\begin{aligned}
(a \cdot \nabla b \mid b)_{\dot{H}^{\frac{1}{2}}} & \leq\|a \cdot \nabla b\|_{L^{2}}\|\nabla b\|_{L^{2}} \\
& \leq\|a\|_{L^{\infty}}\|\nabla b\|_{L^{2}}^{2} .
\end{aligned}
$$

The interpolation inequality between Sobolev norms gives

$$
(a \cdot \nabla b \mid b)_{\dot{H}^{\frac{1}{2}}} \leq\|a\|_{L^{\infty}}\|b\|_{\dot{H}^{\frac{1}{2}}}\|\nabla b\|_{\dot{H}^{\frac{1}{2}}} .
$$

Now let us estimate $(b \cdot \nabla a \mid b)_{\dot{H}^{\frac{1}{2}}}$. Again we use the fact that

$$
(b \cdot \nabla a \mid b)_{\dot{H}^{\frac{1}{2}}} \leq\|b \cdot \nabla a\|_{L^{2}}\|\nabla b\|_{L^{2}} .
$$

Then let us write

$$
\|b \cdot \nabla a\|_{L^{2}}^{2}=\int_{\mathbb{R}^{3}}\left|b\left(x_{h}, x_{3}\right) \nabla a\left(x_{h}, x_{3}\right)\right|^{2} d x_{h} d x_{3} .
$$

Gagliardo-Nirenberg's inequality in the horizontal variable implies that

$$
\forall x_{3} \in \mathbb{R}, \quad\left|b\left(x_{h}, x_{3}\right)\right|^{2} \lesssim\left\|b\left(\cdot, x_{3}\right)\right\|_{\dot{H}_{h}^{\frac{1}{2}}}\left\|\nabla_{h} b\left(\cdot, x_{3}\right)\right\|_{\dot{H}_{h}^{\frac{1}{2}}} \cdot
$$

Let us use the Cauchy-Schwarz inequality; this gives

$$
\begin{aligned}
\|b \cdot \nabla a\|_{L^{2}}^{2} & \leq \int_{\mathbb{R}}\left\|b\left(\cdot, x_{3}\right)\right\|_{\dot{H}_{h}^{\frac{1}{2}}}\left\|\nabla_{h} b\left(\cdot, x_{3}\right)\right\|_{\dot{H}_{h}^{\frac{1}{2}}}\left\|\nabla a\left(\cdot, x_{3}\right)\right\|_{L_{h}^{2}}^{2} d x_{3} \\
& \leq\|\nabla a\|_{L_{v}^{\infty}\left(L_{h}^{2}\right)}^{2} \int_{\mathbb{R}}\left\|b\left(\cdot, x_{3}\right)\right\|_{\dot{H}_{h}^{\frac{1}{2}}}\left\|\nabla_{h} b\left(\cdot, x_{3}\right)\right\|_{\dot{H}_{h}^{\frac{1}{2}}} d x_{3} \\
& \leq\|\nabla a\|_{L_{v}^{\infty} L_{h}^{2}}^{2}\|b\|_{L_{v}^{2} \dot{H}_{h}^{\frac{1}{2}}}\left\|\nabla_{h} b\right\|_{L_{v}^{2} \dot{H}_{h}^{\frac{1}{2}}} .
\end{aligned}
$$

When $s$ is positive, we have, thanks to a Fourier-Plancherel transform in the vertical variable,

$$
\begin{aligned}
\|b\|_{L_{v}^{2}\left(\dot{H}_{h}^{s}\right)} & =\int_{\mathbb{R}} \int_{\mathbb{R}^{2}}\left|\xi_{h}\right|^{2 s}\left|\mathcal{F}_{h} b\left(\xi_{h}, x_{3}\right)\right|^{2} d \xi_{h} d x_{3} \\
& \sim \int_{\mathbb{R}} \int_{\mathbb{R}^{2}}\left|\xi_{h}\right|^{2 s}\left|\widehat{b}\left(\xi_{h}, \xi_{3}\right)\right|^{2} d \xi_{h} d \xi_{3} \\
& \lesssim \int_{\mathbb{R}^{3}}|\xi|^{2 s}|\widehat{b}(\xi)|^{2} d \xi \\
& \lesssim\|b\|_{\dot{H}^{s}}^{2} .
\end{aligned}
$$

This concludes the proof of Lemma 2.3 ,

Conclusion of the proof of Theorem 3. We infer from the above lemma that

$$
\begin{aligned}
\left|\left(R_{\lambda}^{\varepsilon}(t) \cdot \nabla v_{a p p}^{\varepsilon}(t) \mid R_{\lambda}^{\varepsilon}(t)\right)_{\dot{H}^{\frac{1}{2}}}\right|+\left|\left(v_{a p p}^{\varepsilon}(t) \cdot R_{\lambda}^{\varepsilon}(t) \mid R_{\lambda}^{\varepsilon}(t)\right)_{\dot{H}^{\frac{1}{2}}}\right| \\
\leq \frac{1}{4}\left\|\nabla R_{\lambda}^{\varepsilon}(t)\right\|_{\dot{H}^{\frac{1}{2}}}^{2}+C V_{\varepsilon}(t)\left\|R_{\lambda}^{\varepsilon}(t)\right\|_{\dot{H}^{\frac{1}{2}}}^{2} .
\end{aligned}
$$

Together with (2.3), this gives

$$
\begin{aligned}
\frac{1}{2} \frac{d}{d t}\left\|R_{\lambda}^{\varepsilon}(t)\right\|_{\dot{H}^{\frac{1}{2}}}^{2}+\left\|\nabla R_{\lambda}^{\varepsilon}(t)\right\|_{\dot{H}^{\frac{1}{2}}}^{2} & \leq(C-2 \lambda) V_{\varepsilon}(t)\left\|R_{\lambda}^{\varepsilon}(t)\right\|_{\dot{H}^{\frac{1}{2}}}^{2} \\
& +C e^{\lambda I_{0}}\left\|R_{\lambda}^{\varepsilon}(t)\right\|_{\dot{H}^{\frac{1}{2}}}\left\|\nabla R_{\lambda}^{\varepsilon}(t)\right\|_{\dot{H}^{\frac{1}{2}}}^{2}+C\left\|F_{\varepsilon}(t)\right\|_{\dot{H}^{\frac{1}{2}}}^{2} .
\end{aligned}
$$


Choosing $\lambda$ so that $C-2 \lambda$ is negative, we infer that

$$
\frac{d}{d t}\left\|R_{\lambda}^{\varepsilon}(t)\right\|_{\dot{H}^{\frac{1}{2}}}^{2}+\left(1-C e^{\lambda I_{0}}\right)\left\|\nabla R_{\lambda}^{\varepsilon}(t)\right\|_{\dot{H}^{\frac{1}{2}}}^{2} \leq C\left\|F_{\varepsilon}(t)\right\|_{\dot{H}^{\frac{1}{2}}}^{2} .
$$

Since $R^{\varepsilon}(0)=0$, we get, as long as $\left\|R_{\lambda}^{\varepsilon}(t)\right\|_{\dot{H}^{\frac{1}{2}}}$ is less than or equal to $1 / 2 C e^{-\lambda I_{0}}$, that

$$
\left\|R_{\lambda}^{\varepsilon}(t)\right\|_{\dot{H}^{\frac{1}{2}}}^{2}+\frac{1}{2} \int_{0}^{t}\left\|\nabla R_{\lambda}^{\varepsilon}\left(t^{\prime}\right)\right\|_{\dot{H}^{\frac{1}{2}}}^{2} d t^{\prime} \leq C_{v_{0}, w_{0}} \varepsilon^{\frac{1}{3}} .
$$

We therefore obtain that $R^{\varepsilon}$ goes to zero in $L^{\infty}\left(\mathbb{R}^{+} ; \dot{H}^{\frac{1}{2}}\right) \cap L^{2}\left(\mathbb{R}^{+} ; \dot{H}^{\frac{3}{2}}\right)$. This implies that $u^{\varepsilon}$ remains close to $v_{a p p}^{\varepsilon}$ for all times, which in particular implies Theorem 3 .

\section{Estimates on the approximate solution}

In this section we shall prove Lemma 2.1 stated in the previous section. The proof of the lemma is achieved by obtaining estimates on $\underline{v}$, stated in the next lemma, as well as on $\underline{w}^{\varepsilon}$ (see Lemma 3.2 below).

Lemma 3.1. Let $\underline{v}^{h}$ be a solution of the system $\left(N S 2 D_{3}\right)$. Then, for any $s$ greater than -1 and any $\alpha \in \mathbb{N}^{3}$, we have, for any $y_{3}$ in $\mathbb{R}$ and any positive $t$,

$$
\left\|\partial^{\alpha} \underline{v}^{h}\left(t, \cdot, y_{3}\right)\right\|_{\dot{H}_{h}^{s}}^{2}+\int_{0}^{t}\left\|\partial^{\alpha} \nabla_{h} \underline{v}^{h}\left(t^{\prime}, \cdot, y_{3}\right)\right\|_{\dot{H}_{h}^{s}}^{2} d t^{\prime} \leq C_{v_{0}}\left(y_{3}\right)
$$

where $C_{v_{0}}(\cdot)$ belongs to $L^{1} \cap L^{\infty}(\mathbb{R})$ and its norm is controlled by a constant $C_{v_{0}}$.

Proof. For $s=0$ and $\alpha=0$, the estimate is simply the classical $L^{2}$ energy estimate with $y_{3}$ as a parameter: writing $\underline{v}=\left(\underline{v}^{h}, 0\right)$ we have

$$
\left\|\underline{v}\left(t, \cdot, y_{3}\right)\right\|_{L_{h}^{2}}^{2}+2 \int_{0}^{t}\left\|\nabla_{h} \underline{v}\left(t^{\prime}, \cdot, y_{3}\right)\right\|_{L_{h}^{2}}^{2} d t^{\prime}=\left\|\underline{v}_{0}\left(\cdot, y_{3}\right)\right\|_{L_{h}^{2}}^{2} .
$$

In the case where $\alpha=0$, the estimate (i) in Lemma 1.1 of [2] gives, for any $s$ greater than -1 ,

$$
\begin{aligned}
\frac{1}{2} \frac{d}{d t}\left\|\underline{v}\left(t, \cdot, y_{3}\right)\right\|_{\dot{H}_{h}^{s}}^{2}+ & \left\|\nabla_{h} \underline{v}\left(t, \cdot, y_{3}\right)\right\|_{\dot{H}_{h}^{s}}^{2} \\
& \leq C\left\|\nabla_{h} \underline{v}\left(t, \cdot, y_{3}\right)\right\|_{L_{h}^{2}}\left\|\underline{v}\left(t, \cdot, y_{3}\right)\right\|_{\dot{H}_{h}^{s}}\left\|\nabla_{h} \underline{v}\left(t, \cdot, y_{3}\right)\right\|_{\dot{H}_{h}^{s}} .
\end{aligned}
$$

We infer that

$$
\frac{d}{d t}\left\|\underline{v}\left(t, \cdot, y_{3}\right)\right\|_{\dot{H}_{h}^{s}}^{2}+\left\|\nabla_{h} \underline{v}\left(t, \cdot, y_{3}\right)\right\|_{\dot{H}_{h}^{s}}^{2} \leq C\left\|\nabla_{h} \underline{v}\left(t, \cdot, y_{3}\right)\right\|_{L_{h}^{2}}^{2}\left\|\underline{v}\left(t, \cdot, y_{3}\right)\right\|_{\dot{H}_{h}^{s}}^{2} .
$$

Gronwall's lemma ensures that

$$
\begin{aligned}
\left\|\underline{v}\left(t, \cdot, y_{3}\right)\right\|_{\dot{H}_{h}^{s}}^{2}+\int_{0}^{t} \| & \nabla_{h} \underline{v}\left(t^{\prime}, \cdot, y_{3}\right) \|_{\dot{H}_{h}^{s}}^{2} d t^{\prime} \\
& \leq\left\|\underline{v}_{0}\left(\cdot, y_{3}\right)\right\|_{\dot{H}_{h}^{s}}^{2} \exp \left(C \int_{0}^{t}\left\|\nabla_{h} \underline{v}\left(t^{\prime}, \cdot, y_{3}\right)\right\|_{L_{h}^{2}}^{2} d t^{\prime}\right) .
\end{aligned}
$$

The energy estimate (3.1) implies that

$$
\left\|\underline{v}\left(t, \cdot, y_{3}\right)\right\|_{\dot{H}_{h}^{s}}^{2}+\int_{0}^{t}\left\|\nabla_{h} \underline{v}\left(t^{\prime}, \cdot, y_{3}\right)\right\|_{\dot{H}_{h}^{s}}^{2} d t^{\prime} \leq\left\|\underline{v}_{0}\left(\cdot, y_{3}\right)\right\|_{\dot{H}_{h}^{s}}^{2} \exp \left(C\left\|\underline{v}_{0}\right\|_{L_{v}^{\infty} L_{h}^{2}}^{2}\right) .
$$

This proves the lemma in the case where $\alpha=0$. Let us now turn to the general case, which we prove by induction on the length of $\alpha$. It is clear that in the proof we can restrict ourselves to the case where $s \in]-1,1[$. 
Let us assume that, for some $k \in \mathbb{N}$,

$$
\forall s \in]-1,1\left[, \quad \sum_{|\alpha| \leq k}\left(\left\|\partial^{\alpha} \underline{v}\left(t, \cdot, y_{3}\right)\right\|_{\dot{H}_{h}^{s}}^{2}+\int_{0}^{t}\left\|\partial^{\alpha} \nabla_{h} \underline{v}\left(t^{\prime}, \cdot, y_{3}\right)\right\|_{\dot{H}_{h}^{s}}^{2} d t^{\prime}\right) \leq C_{k, v_{0}}\left(y_{3}\right),\right.
$$

with $C_{k, v_{0}}(\cdot) \in L^{1} \cap L^{\infty}(\mathbb{R})$.

Thanks to the Leibnitz formula we have, for $|\alpha| \leq k+1$,

$$
\partial_{t} \partial^{\alpha} \underline{v}^{h}+\underline{v}^{h} \cdot \nabla_{h} \partial^{\alpha} \underline{v}^{h}-\Delta_{h} \partial^{\alpha} \underline{v}^{h}=-\nabla_{h} p_{\alpha}-\sum_{\substack{\beta \leq \alpha \\ \beta \neq \alpha}} C_{\alpha}^{\beta} \partial^{\alpha-\beta} \underline{v}^{h} \cdot \nabla_{h} \partial^{\beta} \underline{v}^{h} .
$$

Performing an $\dot{H}_{h}^{s}$ energy estimate in the horizontal variable and using the estimate (2.2) in the case where $d=2$ gives

$$
\begin{gathered}
\frac{1}{2} \frac{d}{d t}\left\|\partial^{\alpha} \underline{v}\left(t, \cdot, y_{3}\right)\right\|_{\dot{H}_{h}^{s}}^{2}+\left\|\nabla_{h} \partial^{\alpha} \underline{v}\left(t, \cdot, y_{3}\right)\right\|_{\dot{H}_{h}^{s}}^{2} \\
\leq C\left\|\nabla_{h} \underline{v}\left(t, \cdot, y_{3}\right)\right\|_{L_{h}^{2}}\left\|\partial^{\alpha} \underline{v}\left(t, \cdot, y_{3}\right)\right\|_{\dot{H}_{h}^{s}}\left\|\nabla_{h} \partial^{\alpha} \underline{v}\left(t, \cdot, y_{3}\right)\right\|_{\dot{H}_{h}^{s}} \\
+C_{\alpha} \sum_{\substack{\beta \leq \alpha \\
\beta \neq \alpha}}\left|\left(\partial^{\alpha-\beta} \underline{v}^{h}\left(t, \cdot, y_{3}\right) \cdot \nabla_{h} \partial^{\beta} \underline{v}^{h}\left(t, \cdot, y_{3}\right) \mid \partial^{\alpha} \underline{v} t, \cdot h\left(t, \cdot, y_{3}\right)\right)_{\dot{H}_{h}^{s}}\right| \cdot
\end{gathered}
$$

To estimate the last term, we shall treat separately the cases $|\beta|=0$ and $|\beta| \neq 0$. In the former case, we notice first that when $s=0$, laws of products for Sobolev spaces in $\mathbb{R}^{2}$ give

$$
\begin{aligned}
\left(\partial^{\alpha} \underline{v}^{h}\left(t, \cdot, y_{3}\right)\right. & \left.\cdot \nabla_{h} \underline{v}^{h}\left(t, \cdot, y_{3}\right) \mid \partial^{\alpha} \underline{v}^{h}\left(t, \cdot, y_{3}\right)\right)_{L_{h}^{2}} \\
& \lesssim\left\|\partial^{\alpha} \underline{v}^{h}\left(t, \cdot, y_{3}\right)\right\|_{\dot{H}^{\frac{1}{2}}}^{2}\left\|\nabla_{h} \underline{v}^{h}\left(t, \cdot, y_{3}\right)\right\|_{L^{2}} \\
& \lesssim\left\|\partial^{\alpha} \underline{v}^{h}\left(t, \cdot, y_{3}\right)\right\|_{L_{h}^{2}}\left\|\nabla_{h} \partial^{\alpha} \underline{v}^{h}\left(t, \cdot, y_{3}\right)\right\|_{L_{h}^{2}}\left\|\nabla_{h} \underline{v}^{h}\left(t, \cdot, y_{3}\right)\right\|_{L_{h}^{2}} .
\end{aligned}
$$

If $s>0$, then again laws of products for Sobolev spaces in $\mathbb{R}^{2}$ give, for $\left.s \in\right] 0,1$,

$\left|\left(\partial^{\alpha} \underline{v}^{h}\left(t, \cdot, y_{3}\right) \cdot \nabla_{h} \underline{v}^{h}\left(t, \cdot, y_{3}\right) \mid \partial^{\alpha} \underline{v}\left(t, \cdot, y_{3}\right)\right)_{\dot{H}_{h}^{s}}\right| \lesssim\left\|\partial^{\alpha} \underline{v}^{h}\right\|_{\dot{H}_{h}^{s}}\left\|\nabla_{h} \underline{v}^{h}\right\|_{L_{h}^{2}}\left\|\nabla_{h} \partial^{\alpha} \underline{v}^{h}\right\|_{\dot{H}_{h}^{s}}$,

whereas if $-1<s<0$,

$\left|\left(\partial^{\alpha} \underline{v}^{h}\left(t, \cdot, y_{3}\right) \cdot \nabla_{h} \underline{v}^{h}\left(t, \cdot, y_{3}\right) \mid \partial^{\alpha} \underline{v}\left(t, \cdot, y_{3}\right)\right)_{\dot{H}_{h}^{s}}\right| \lesssim\left\|\nabla_{h} \partial^{\alpha} \underline{v}^{h}\right\|_{\dot{H}_{h}^{s}}\left\|\nabla_{h} \underline{v}^{h}\right\|_{L_{h}^{2}}\left\|\partial^{\alpha} \underline{v}^{h}\right\|_{\dot{H}_{h}^{s}}$.

So in any case we have

$$
\begin{aligned}
\mid\left(\partial^{\alpha} \underline{v}^{h}\left(t, \cdot, y_{3}\right) \cdot \nabla_{h} \underline{v}^{h}\left(t, \cdot, y_{3}\right) \mid\right. & \left.\partial^{\alpha} \underline{v}\left(t, \cdot, y_{3}\right)\right)_{\dot{H}_{h}^{s}} \mid \\
& \leq \frac{1}{4}\left\|\nabla_{h} \partial^{\alpha} \underline{v}^{h}\right\|_{\dot{H}_{h}^{s}}^{2}+C\left\|\nabla_{h} \underline{v}^{h}\right\|_{L_{h}^{2}}^{2}\left\|\partial^{\alpha} \underline{v}^{h}\right\|_{\dot{H}_{h}^{s}}^{2}
\end{aligned}
$$

Now let us consider the case $|\beta| \neq 0$. As the horizontal divergence of $\underline{v}$ is identically 0 , we have

$$
\begin{aligned}
\left|\left(\partial^{\alpha-\beta} \underline{v}^{h} \cdot \nabla_{h} \partial^{\beta} \underline{v}^{h} \mid \partial^{\alpha} \underline{v}\left(t, \cdot, y_{3}\right)\right)_{\dot{H}_{h}^{s}}\right| & \\
& \leq\left\|\partial^{\alpha-\beta} \underline{v}^{h}\left(t, \cdot, y_{3}\right) \otimes \partial^{\beta} \underline{v}^{h}\left(t, \cdot, y_{3}\right)\right\|_{\dot{H}_{h}^{s}}\left\|\nabla_{h} \partial^{\alpha} \underline{v}\left(t, \cdot, y_{3}\right)\right\|_{\dot{H}_{h}^{s}} .
\end{aligned}
$$

Laws of products for Sobolev spaces in $\mathbb{R}^{2}$ give, for $\left.s \in\right]-1,1$,

$$
\left\|\partial^{\alpha-\beta} \underline{v}^{h} \otimes \partial^{\beta} \underline{v}^{h}\right\|_{\dot{H}_{h}^{s}} \leq C\left\|\partial^{\alpha-\beta} \underline{v}^{h}\right\|_{\dot{H}_{h}^{s^{\prime}}}\left\|\partial^{\beta} \nabla_{h} \underline{v}^{h}\right\|_{\dot{H}_{h}^{s-s^{\prime}}},
$$

where $s^{\prime}$ is chosen so that $s<s^{\prime}<1$. 
Finally we deduce that

$$
\begin{aligned}
& \frac{d}{d t}\left\|\partial^{\alpha} \underline{v}\left(t, \cdot, y_{3}\right)\right\|_{\dot{H}_{h}^{s}}^{2}+\left\|\nabla_{h} \partial^{\alpha} \underline{v}\left(t, \cdot, y_{3}\right)\right\|_{\dot{H}_{h}^{s}}^{2} \leq C\left\|\nabla_{h} \underline{v}\right\|_{L_{h}^{2}}^{2}\left\|\partial^{\alpha} \underline{v}\right\|_{\dot{H}_{h}^{s}}^{2} \\
&+C_{\alpha} \sum_{\substack{\beta \leq \alpha \\
\beta \notin\{0, \alpha\}}}\left\|\partial^{\alpha-\beta} \underline{v}\left(t, \cdot, y_{3}\right)\right\|_{\dot{H}_{h}^{s^{\prime}}}\left\|\partial^{\beta} \nabla_{h} \underline{v}\left(t, \cdot, y_{3}\right)\right\|_{\dot{H}_{h}^{s-s^{\prime}}}\left\|\nabla_{h} \partial^{\alpha} \underline{v}\left(t, \cdot, y_{3}\right)\right\|_{\dot{H}_{h}^{s}} .
\end{aligned}
$$

Gronwall's lemma together with the induction hypothesis (3.2) implies that

$$
\begin{aligned}
& \sum_{|\alpha|=k+1}\left(\left\|\partial^{\alpha} \underline{v}\left(t, \cdot, y_{3}\right)\right\|_{\dot{H}_{h}^{s}}^{2}+\int_{0}^{t}\left\|\partial^{\alpha} \nabla_{h} \underline{v}\left(t^{\prime}, \cdot, y_{3}\right)\right\|_{\dot{H}_{h}^{s}}^{2} d t^{\prime}\right) \\
\lesssim & \left(\sum_{|\alpha|=k+1}\left\|\partial^{\alpha} \underline{v}_{0}\left(\cdot, y_{3}\right)\right\|_{\dot{H}_{h}^{s}}^{2}+C_{k, v_{0}}\left(y_{3}\right)\right) \exp \left(C_{k} \int_{0}^{t}\left\|\nabla_{h} \underline{v}\left(t^{\prime}, \cdot, y_{3}\right)\right\|_{L_{h}^{2}}^{2} d t^{\prime}\right) .
\end{aligned}
$$

The $L^{2}$ energy estimate (3.1) allows us to conclude the proof of Lemma 3.1 ,

From this lemma, we deduce the following corollary.

Corollary 3.1. Let $\underline{v}^{h}$ be a solution of the system $\left(N S 2 D_{3}\right)$. Then, for any nonnegative $\sigma$, we have

$$
\left\|\underline{v}^{h}\right\|_{L^{2}\left(\mathbb{R}^{+} ; \dot{H}^{\sigma}\left(\mathbb{R}^{3}\right)\right)} \leq C_{v_{0}} \quad \text { and } \quad\left\|\partial^{\alpha} \underline{v}^{h}\right\|_{L^{2}\left(\mathbb{R}^{+} ; L_{v}^{\infty} \dot{H}_{h}^{\sigma}\right)} \leq C_{v_{0}} .
$$

Proof. To start with, let us assume $\sigma>0$. Lemma 3.1 applied with $s=\sigma-1$ implies that

$$
\forall \sigma>0, \forall \alpha \in \mathbb{N},\left\|\partial^{\alpha} \underline{v}^{h}\right\|_{L^{2}\left(\mathbb{R}^{+} ; L_{v}^{2} \dot{H}_{h}^{\sigma}\right)} \leq C_{v_{0}}
$$

Then, for any nonnegative $\sigma$, we have

$$
\begin{aligned}
\left\|\partial^{\alpha} \underline{v}\left(t, \cdot, y_{3}\right)\right\|_{\dot{H}_{h}^{\sigma}}^{2} & =2 \int_{-\infty}^{y_{3}}\left(\partial_{3} \partial^{\alpha} \underline{v}\left(t, \cdot, y_{3}^{\prime}\right) \mid \partial^{\alpha} \underline{v}\left(t, \cdot, y_{3}^{\prime}\right)_{\dot{H}_{h}^{\sigma}} d y_{3}^{\prime}\right. \\
& \leq 2\left\|\partial_{3} \partial^{\alpha} \underline{v}^{h}(t, \cdot)\right\|_{L_{v}^{2} \dot{H}_{h}^{\sigma}}\left\|\partial^{\alpha} \underline{v}^{h}(t, \cdot)\right\|_{L_{v}^{2} \dot{H}_{h}^{\sigma}}
\end{aligned}
$$

By the Cauchy-Schwarz inequality, we have

$$
\begin{aligned}
\forall \sigma \geq 0, & \forall \alpha \in \mathbb{N}, \\
& \left\|\partial^{\alpha} \underline{v}^{h}\right\|_{L^{2}\left(\mathbb{R}^{+} ; L_{v}^{\infty} \dot{H}_{h}^{\sigma}\right)} \leq\left\|\partial_{3} \partial^{\alpha} \underline{v}^{h}\right\|_{L^{2}\left(\mathbb{R}^{+} ; L_{v}^{\infty} \dot{H}_{h}^{\sigma}\right)}^{\frac{1}{2}}\left\|\partial^{\alpha} \underline{v}^{h}\right\|_{L^{2}\left(\mathbb{R}^{+} ; L_{v}^{\infty} \dot{H}_{h}^{\sigma}\right)}^{\frac{1}{2}} .
\end{aligned}
$$

From (3.3), we infer that

$$
\forall \sigma>0, \forall \alpha \in \mathbb{N},\left\|\partial^{\alpha} \underline{v}^{h}\right\|_{L^{2}\left(\mathbb{R}^{+} ; L_{v}^{\infty} \dot{H}_{h}^{\sigma}\right)} \leq C_{v_{0}} .
$$

Now, by interpolation, it is enough to prove the first inequality with $\sigma=0$. The system $\left(N S 2 D_{3}\right)$ can be written as

$$
\left\{\begin{array}{c}
\partial_{t} \underline{v}-\Delta_{h} \underline{v}=f \\
\underline{v}_{\mid t=0}=\underline{v}_{0}\left(\cdot, y_{3}\right)
\end{array} \quad \text { with } \quad f \stackrel{\text { def }}{=} \sum_{1 \leq j, k \leq 2} Q_{j, k}(D)\left(\underline{v}^{j} \underline{v}^{k}\right),\right.
$$


where $Q_{j, k}$ are homogenenous smooth Fourier multipliers of order 1. By Sobolev embeddings in $\mathbb{R}^{2}$, we get, for any $y_{3}$ in $\mathbb{R}$,

$$
\begin{aligned}
\left\|\underline{v}\left(\cdot, y_{3}\right)\right\|_{L^{2}\left(\mathbb{R}^{+} \times \mathbb{R}^{2}\right)} \leq & \left\|\underline{v}_{0}\left(\cdot, y_{3}\right)\right\|_{\dot{H}_{h}^{-1}}+\left\|f\left(\cdot, y_{3}\right)\right\|_{L^{1}\left(\mathbb{R}^{+} ; \dot{H}_{h}^{-1}\right)} \\
\leq & \left\|\underline{v}_{0}\left(\cdot, y_{3}\right)\right\|_{\dot{H}_{h}^{-1}}+C\left\|\underline{v}\left(\cdot, y_{3}\right)\right\|_{L^{2}\left(\mathbb{R}^{+} ; \dot{H}_{h}^{\frac{1}{2}}\right)}^{2} \\
\leq & \left\|\underline{v}_{0}\left(\cdot, y_{3}\right)\right\|_{\dot{H}_{h}^{-1}} \\
& +C\left\|\underline{v}\left(\cdot, y_{3}\right)\right\|_{L^{2}\left(\mathbb{R}^{+} ; \dot{H}_{h}^{\frac{1}{2}}\right)} \sup _{y_{3}}\left\|\underline{v}\left(\cdot, y_{3}\right)\right\|_{L^{2}\left(\mathbb{R}^{+} ; \dot{H}_{h}^{\frac{1}{2}}\right.} .
\end{aligned}
$$

As $\sup _{y_{3}}\left\|\underline{v}\left(\cdot, y_{3}\right)\right\|_{L^{2}\left(\mathbb{R}^{+} ; \dot{H}_{h}^{\frac{1}{2}}\right)} \leq\|\underline{v}\|_{L^{2}\left(\mathbb{R}^{+} ; L_{v}^{\infty} \dot{H}_{h}^{\frac{1}{2}}\right)}$, we infer from (3.4) that

$$
\|\underline{v}\|_{L^{2}\left(\mathbb{R}^{+} \times \mathbb{R}^{3}\right)}^{2} \lesssim\left\|\underline{v}_{0}\right\|_{L_{v}^{2} \dot{H}_{h}^{-1}}^{2}+C_{v_{0}} \| \underline{v}_{L^{2}\left(\mathbb{R}^{+} ; L_{v}^{2} \dot{H}_{h}^{\frac{1}{2}}\right)}^{2} \leq C_{v_{0}} .
$$

The corollary is proved.

Finally we have the following estimate on $\underline{w}^{\varepsilon}$.

Lemma 3.2. Let $\underline{w}^{\varepsilon}$ be a solution of the system $\left(T_{\underline{v}}^{\varepsilon}\right)$. Then, for any s greater than -1 , any $\alpha \in \mathbb{N}^{3}$ and any positive $t$, we have

$$
\left\|\partial^{\alpha} \underline{w}^{\varepsilon}(t, \cdot)\right\|_{L_{v}^{2} \dot{H}_{h}^{s}}^{2}+\int_{0}^{t}\left\|\partial^{\alpha} \nabla_{h} \underline{w}^{\varepsilon}\left(t^{\prime}, \cdot\right)\right\|_{L_{v}^{2} \dot{H}_{h}^{s}}^{2} d t^{\prime} \leq C_{v_{0}, w_{0}} .
$$

Proof. We shall only sketch the proof, as it is very similar to the proof of Lemma 3.1 which was carried out above. The only difference is that the horizontal divergence of $\underline{w}$ does not vanish identically, but this will not change the estimates by very much. We shall only present the proof in the case where $\alpha=0$ and $-1<s<1$, and leave the general case to the reader. Using Lemma 1.1 of [2] we have, for any $y_{3}$ in $\mathbb{R}$,

$$
\begin{aligned}
\left(\underline{v}^{h}\left(t, \cdot, y_{3}\right) \cdot \nabla_{h} \underline{w}^{\varepsilon}\left(t, \cdot, y_{3}\right) \mid \underline{w}^{\varepsilon}\left(t, \cdot, y_{3}\right)\right)_{\dot{H}_{h}^{s}} & \\
& \leq C\left\|\nabla_{h} \underline{v}\left(t, \cdot, y_{3}\right)\right\|_{L_{h}^{2}}\left\|\nabla_{h} \underline{w}^{\varepsilon}\left(t, \cdot, y_{3}\right)\right\|_{\dot{H}_{h}^{s}}\left\|\underline{w}^{\varepsilon}\left(t, \cdot, y_{3}\right)\right\|_{\dot{H}_{h}^{s}} .
\end{aligned}
$$

Thus we get

$$
\begin{gathered}
\frac{1}{2} \frac{d}{d t}\left\|\underline{w}^{\varepsilon}(t)\right\|_{L_{v}^{2} \dot{H}_{h}^{s}}^{2}+\left\|\nabla_{h} \underline{w}^{\varepsilon}(t)\right\|_{L_{v}^{2} \dot{H}_{h}^{s}}^{2} \\
\leq \frac{1}{4}\left\|\nabla_{h} \underline{w}^{\varepsilon}(t)\right\|_{L_{v}^{2} \dot{H}_{h}^{s}}^{2}+C\left\|\nabla_{h} \underline{v}^{h}(t)\right\|_{L_{v}^{\infty} L_{h}^{2}}^{2}\left\|\underline{w}^{\varepsilon}(t)\right\|_{L_{v}^{2} \dot{H}_{h}^{s}}^{2} \\
-\varepsilon^{2} \int_{\mathbb{R}}\left(\partial_{3} \underline{p}_{1}\left(t, \cdot, y_{3}\right) \mid \underline{w}^{\varepsilon, 3}\left(t, \cdot, y_{3}\right)\right)_{\dot{H}_{h}^{s}} d y_{3}-\int_{\mathbb{R}}\left(\nabla^{h} \underline{p}_{1}\left(t, \cdot, y_{3}\right) \mid \underline{w}^{\varepsilon, h}\left(t, \cdot, y_{3}\right)\right)_{\dot{H}_{h}^{s}} d y_{3} .
\end{gathered}
$$

Using integration by parts we have, thanks to the divergence free condition on $\underline{w}^{\varepsilon}$,

$$
\begin{aligned}
-\int_{\mathbb{R}}\left(\partial_{3} \underline{p}_{1}\left(t, \cdot, y_{3}\right) \mid \underline{w}^{\varepsilon, 3}\left(t, \cdot, y_{3}\right)\right)_{\dot{H}_{h}^{s}} d y_{3} & =\int_{\mathbb{R}}\left(\underline{p}_{1}\left(t, \cdot, y_{3}\right) \mid \partial_{3} \underline{w}^{\varepsilon, 3}\left(t, \cdot, y_{3}\right)\right)_{\dot{H}_{h}^{s}} d y_{3} \\
& =-\int_{\mathbb{R}}\left(\underline{p}_{1}\left(t, \cdot, y_{3}\right) \mid \operatorname{div}_{h} \underline{w}^{\varepsilon, h}\left(t, \cdot, y_{3}\right)\right)_{\dot{H}_{h}^{s}} d y_{3} .
\end{aligned}
$$

By definition of the inner product of $\dot{H}_{h}^{s}$, we get

$$
-\int_{\mathbb{R}}\left(\partial_{3} \underline{p}_{1}\left(t, \cdot, y_{3}\right) \mid \underline{w}^{\varepsilon, 3}\left(t, \cdot, y_{3}\right)\right)_{\dot{H}_{h}^{s}} d y_{3}=\int_{\mathbb{R}}\left(\nabla_{h} \underline{p}_{1}\left(t, \cdot, y_{3}\right) \mid \underline{w}^{\varepsilon, h}\left(t, \cdot, y_{3}\right)\right)_{\dot{H}_{h}^{s}} d y_{3} .
$$


Thus we have

$$
\begin{gathered}
\frac{1}{2} \frac{d}{d t}\left\|\underline{w}^{\varepsilon}(t)\right\|_{L_{v}^{2} \dot{H}_{h}^{s}}^{2}+\left\|\nabla_{h} \underline{w}^{\varepsilon}(t)\right\|_{L_{v}^{2} \dot{H}_{h}^{s}}^{2} \leq \frac{1}{4}\left\|\nabla_{h} \underline{w}^{\varepsilon}(t)\right\|_{L_{v}^{2} \dot{H}_{h}^{s}}^{2} \\
+C\left\|\nabla_{h} \underline{v}^{h}(t)\right\|_{L_{v}^{\infty} L_{h}^{2}}^{2}\left\|\underline{w}^{\varepsilon}(t)\right\|_{L_{v}^{2} \dot{H}_{h}^{s}}^{2}-\left(1-\varepsilon^{2}\right) \int_{\mathbb{R}}\left(\nabla_{h} \underline{p}_{1}\left(t, \cdot, y_{3}\right) \mid \underline{w}^{\varepsilon, h}\left(t, \cdot, y_{3}\right)\right)_{\dot{H}_{h}^{s}} d y_{3} .
\end{gathered}
$$

Now we notice that

$$
-\left(\varepsilon^{2} \partial_{3}^{2}+\Delta_{h}\right) \underline{p}_{1}=\operatorname{div}\left(\underline{v}^{h} \cdot \nabla_{h} \underline{w}^{\varepsilon}\right)=\operatorname{div} \sum_{j=1}^{2} \partial_{j}\left(\underline{v}^{j} \underline{w}^{\varepsilon}\right),
$$

which can be written in the simpler form

$$
-\left(\varepsilon^{2} \partial_{3}^{2}+\Delta_{h}\right) \underline{p}_{1}=\operatorname{div}_{h} N^{h}
$$

with $N^{h}=\underline{v}^{h} \cdot \nabla_{h} \underline{w}^{\varepsilon, h}+\partial_{3}\left(\underline{w}^{\varepsilon, 3} \underline{v}^{h}\right)$. It is easy to check that for any $\sigma \in \mathbb{R}$,

$$
\left\|\nabla_{h} \underline{p}_{1}\right\|_{L_{v}^{2} \dot{H}_{h}^{\sigma}} \lesssim\left\|N^{h}\right\|_{L_{v}^{2} \dot{H}_{h}^{\sigma}}
$$

simply by noticing that

$$
\begin{aligned}
\left\|\nabla_{h} \underline{p}_{1}\right\|_{L_{v}^{2} \dot{H}_{h}^{\sigma}}^{2} & \sim \int\left|\xi_{h}\right|^{2 \sigma+2}\left|\widehat{\underline{p}}_{1}(\xi)\right|^{2} d \xi \\
& \sim \int\left|\xi_{h}\right|^{2 \sigma+4}\left|\widehat{N}^{h}(\xi)\right|^{2} \frac{d \xi}{\left(\left|\xi_{h}\right|^{2}+\varepsilon^{2}\left|\xi_{3}\right|^{2}\right)^{2}} \\
& \lesssim\left\|N^{h}\right\|_{L_{v}^{2} \dot{H}_{h}^{\sigma}}^{2} .
\end{aligned}
$$

We infer from (3.5) that

$$
\begin{aligned}
\left\|\nabla_{h} \underline{p}_{1}\right\|_{L_{v}^{2} \dot{H}_{h}^{\sigma}} & \leq\left\|\underline{v}^{h} \cdot \nabla_{h} \underline{w}^{\varepsilon, h}\right\|_{L_{v}^{2} \dot{H}_{h}^{\sigma}}+\left\|\partial_{3}\left(\underline{w}^{\varepsilon, 3} \underline{v}^{h}\right)\right\|_{L_{v}^{2} \dot{H}_{h}^{\sigma}} \\
& \leq\left\|\underline{v}^{h} \cdot \nabla_{h} \underline{w}^{\varepsilon}\right\|_{L_{v}^{2} \dot{H}_{h}^{\sigma}}+\left\|\underline{w}^{\varepsilon, 3} \partial_{3} \underline{v}\right\|_{L_{v}^{2} \dot{H}_{h}^{\sigma}}+\left\|\underline{v} \operatorname{div}_{h} \underline{w}^{\varepsilon, h}\right\|_{L_{v}^{2} \dot{H}_{h}^{\sigma}} .
\end{aligned}
$$

We claim that for all $-1<s<1$,

$$
\begin{aligned}
& \mathcal{I}_{h} \stackrel{\text { def }}{=} \int_{\mathbb{R}}\left|\left(\nabla_{h} \underline{p}_{1}\left(t, \cdot, y_{3}\right) \mid \underline{w}^{\varepsilon}\left(t, \cdot, y_{3}\right)\right)\right|_{\dot{H}_{h}^{s}} d y_{3} \\
& \quad \leq \frac{1}{4}\left\|\nabla_{h} \underline{w}^{\varepsilon}\right\|_{L_{v}^{2} \dot{H}_{h}^{s}}^{2}+C\left\|\underline{w}^{\varepsilon}\right\|_{L_{v}^{2} \dot{H}_{h}^{s}}^{2}\left\|\nabla \underline{v}^{h}\right\|_{L_{v}^{\infty} L_{h}^{2}}^{2}\left(1+\left\|\underline{v}^{h}\right\|_{L_{v}^{\infty} L_{h}^{2}}^{2}\right)
\end{aligned}
$$

Let us prove the claim. Suppose first that $s=0$. Then a product law gives

$$
\begin{aligned}
\mathcal{I}_{h} & \leq\left\|\underline{w}^{\varepsilon}\right\|_{L_{v}^{2} \dot{H}_{h}^{\frac{1}{2}}}\left\|\nabla_{h} \underline{p}_{1}\right\|_{L_{v}^{2} \dot{H}_{h}^{-\frac{1}{2}}} \\
& \lesssim\left\|\underline{w}^{\varepsilon}\right\|_{L_{v}^{2} \dot{H}_{h}^{\frac{1}{2}}}\left\|\underline{v}^{h}\right\|_{L_{v}^{\infty} \dot{H}_{h}^{\frac{1}{2}}}\left\|\nabla_{h} \underline{w}^{\varepsilon}\right\|_{L^{2}\left(\mathbb{R}^{3}\right)}+\left\|\underline{w}^{\varepsilon}\right\|_{L_{v}^{2} \dot{H}_{h}^{\frac{1}{2}}}^{2}\left\|\partial_{3} \underline{v}\right\|_{L_{v}^{\infty} L_{h}^{2}} .
\end{aligned}
$$

By interpolation, we get

$$
\begin{aligned}
\mathcal{I}_{h} \leq & \left\|\nabla_{h} \underline{w}^{\varepsilon}\right\|_{L^{2}\left(\mathbb{R}^{3}\right)}^{\frac{3}{2}}\left\|\underline{w}^{\varepsilon}\right\|_{L^{2}\left(\mathbb{R}^{3}\right)}^{\frac{1}{2}}\left\|\underline{v}^{h}\right\|_{L_{v}^{\infty} L_{h}^{2}}^{\frac{1}{2}}\left\|\nabla_{h} \underline{v}^{h}\right\|_{L_{v}^{\infty} L_{h}^{2}}^{\frac{1}{2}} \\
& +\left\|\underline{w}^{\varepsilon}\right\|_{L^{2}\left(\mathbb{R}^{3}\right)}\left\|\nabla_{h} \underline{w}^{\varepsilon}\right\|_{L^{2}\left(\mathbb{R}^{3}\right)}\left\|\partial_{3} \underline{v}\right\|_{L_{v}^{\infty} L_{h}^{2}} .
\end{aligned}
$$

The claim in the case $s=0$ follows from a convexity inequality, which gives

$$
\mathcal{I}_{h} \leq \frac{1}{4}\left\|\nabla_{h} \underline{w}^{\varepsilon}\right\|_{L^{2}\left(\mathbb{R}^{3}\right)}^{2}+C\left\|\underline{w}^{\varepsilon}\right\|_{L^{2}\left(\mathbb{R}^{3}\right)}^{2}\left(1+\|\underline{v}\|_{L_{v}^{\infty} L_{h}^{2}}^{2}\|\nabla \underline{v}\|_{L_{v}^{\infty} L_{h}^{2}}^{2} .\right.
$$

In the case where $0<s<1$, we use the product rule

$$
\left\|\nabla_{h} \underline{p}_{1}\right\|_{L_{v}^{2} \dot{H}_{h}^{s-1}} \lesssim\left\|\underline{v}^{h}\right\|_{L_{v}^{\infty} \dot{H}_{h}^{\frac{1}{2}}}\left\|\nabla_{h} \underline{w}^{\varepsilon}\right\|_{L_{v}^{2} \dot{H}_{h}^{s-\frac{1}{2}}}+\left\|\underline{w}^{\varepsilon}\right\|_{L_{v}^{2} \dot{H}_{h}^{s}}\|\nabla \underline{v}\|_{L_{v}^{\infty} L_{h}^{2}}
$$


along with the fact that

$$
\mathcal{I}_{h} \leq\left\|\nabla_{h} \underline{p}_{1}\right\|_{L_{v}^{2} \dot{H}_{h}^{s-1}}\left\|\nabla_{h} \underline{w}^{\varepsilon}\right\|_{L_{v}^{2} \dot{H}_{h}^{s}} .
$$

Finally in the case where $-1<s<0$, we write

$$
\begin{aligned}
\mathcal{I}_{h} & \leq\left\|\underline{w}^{\varepsilon} \cdot \nabla \underline{v}^{h}\right\|_{L_{v}^{2} \dot{H}_{h}^{s}}\left\|\underline{w}^{\varepsilon}\right\|_{L_{v}^{2} \dot{H}_{h}^{s}}+\left\|\underline{w}^{\varepsilon} \cdot \underline{v}^{h}\right\|_{L_{v}^{2} \dot{H}_{h}^{s}}\left\|\nabla_{h} \underline{w}^{\varepsilon}\right\|_{L_{v}^{2} \dot{H}_{h}^{s}} \\
& \lesssim\left\|\nabla \underline{v}^{h}\right\|_{L_{v}^{\infty} L_{h}^{2}}\left\|\underline{w}^{\varepsilon}\right\|_{L_{v}^{2} \dot{H}_{h}^{s}}\left\|\nabla_{h} \underline{w}^{\varepsilon}\right\|_{L_{v}^{2} \dot{H}_{h}^{s}}+\left\|\underline{v}^{h}\right\|_{L_{v}^{\infty} \dot{H}_{h}^{\frac{1}{2}}}\left\|\underline{w}^{\varepsilon}\right\|_{L_{v}^{2} \dot{H}_{h}^{s+\frac{1}{2}}}\left\|\nabla_{h} \underline{w}^{\varepsilon}\right\|_{L_{v}^{2} \dot{H}_{h}^{s}} .
\end{aligned}
$$

The claim (3.6) follows by interpolation.

Using this result we obtain that

$\frac{d}{d t}\left\|\underline{w}^{\varepsilon}(t)\right\|_{L_{v}^{2} \dot{H}_{h}^{s}}^{2}+\left\|\nabla_{h} \underline{w}^{\varepsilon}(t)\right\|_{L_{v}^{2} \dot{H}_{h}^{s}}^{2} \lesssim\left\|\nabla \underline{v}^{h}(t)\right\|_{L_{v}^{\infty} L_{h}^{2}}^{2}\left\|\underline{w}^{\varepsilon}(t)\right\|_{L_{v}^{2} \dot{H}_{h}^{s}}^{2}\left(1+\left\|\underline{v}^{h}(t)\right\|_{L_{v}^{\infty} L_{h}^{2}}^{2}\right)$

and we conclude by a Gronwall lemma. Indeed we get that

$$
\begin{aligned}
\left\|\underline{w}^{\varepsilon}(t)\right\|_{L_{v}^{2} \dot{H}_{h}^{s}}^{2}+\int_{0}^{t} \| & \nabla_{h} \underline{w}^{\varepsilon}\left(t^{\prime}\right)\left\|_{L_{v}^{2} \dot{H}_{h}^{s}}^{2} d t^{\prime} \leq\right\| w_{0} \|_{L_{v}^{2} \dot{H}_{h}^{s}}^{2} \\
& \times \exp \left(C \int_{0}^{t}\left\|\nabla \underline{v}^{h}\left(t^{\prime}\right)\right\|_{L_{v}^{\infty} L_{h}^{2}}^{2}\left(1+\left\|\underline{v}^{h}\left(t^{\prime}\right)\right\|_{L_{v}^{\infty} L_{h}^{2}}^{2}\right) d t^{\prime}\right) .
\end{aligned}
$$

But by the basic energy estimate (3.1), we have

$$
\left\|\underline{v}^{h}(t)\right\|_{L_{v}^{\infty} L_{h}^{2}} \leq\left\|v_{0}\right\|_{L_{v}^{\infty} L_{h}^{2}} .
$$

Moreover, Corollary 3.1 implies that

$$
\nabla \underline{v}^{h} \in L^{2}\left(\mathbb{R}^{+} ; L_{v}^{\infty} L_{h}^{2}\right),
$$

so Lemma 3.2 is proved in the case $\alpha=0$. The case where $\alpha$ is positive is an easy adaptation of the proof of Lemma 3.1, it is left to the reader.

Clearly Lemmas 3.1 and 3.2 allow us to obtain Lemma 2.1 stated in the previous section.

\section{The ESTIMATE OF THE ERROR TERM}

In this section we shall prove Lemma 2.2 stated above. We need to write down precisely the equation satisfied by the remainder term $R^{\varepsilon}$ and check that the forcing terms appearing in the equation can be made small.

Let us recall that

$$
\begin{aligned}
& v_{a p p}^{\varepsilon}(t, x)=\left(\left(\underline{v}^{h}, 0\right)+\varepsilon\left(\underline{w}^{\varepsilon, h}, \varepsilon^{-1} \underline{w}^{\varepsilon, 3}\right)\right)\left(t, x_{h}, \varepsilon x_{3}\right) \quad \text { and } \\
& p_{a p p}^{\varepsilon}(t, x)=\left(\underline{p}_{0}+\varepsilon \underline{p}_{1}\right)\left(t, x_{h}, \varepsilon x_{3}\right) .
\end{aligned}
$$

It is an easy computation to see that

$$
\begin{aligned}
\left(\partial_{t} v_{a p p}^{\varepsilon}+v_{a p p}^{\varepsilon} \cdot \nabla v_{a p p}^{\varepsilon}-\Delta v_{a p p}^{\varepsilon}\right)\left(t, x_{h}, x_{3}\right) \\
=\left(\partial_{t} \underline{v}^{h}+\underline{v}^{h} \cdot \nabla_{h} \underline{v}^{h}-\Delta_{h} \underline{v}^{h}, 0\right)\left(t, x_{h}, \varepsilon x_{3}\right) \\
\quad+\varepsilon\left(\partial_{t} \underline{w}^{\varepsilon, h}+\underline{v}^{h} \cdot \nabla_{h} \underline{w}^{\varepsilon, h}-\Delta_{h} \underline{w}^{\varepsilon, h}-\varepsilon^{2} \partial_{3}^{2} \underline{w}^{\varepsilon, h}, 0\right)\left(t, x_{h}, \varepsilon x_{3}\right) \\
\quad+\left(0, \partial_{t} \underline{w}^{\varepsilon, 3}+\underline{v}^{h} \cdot \nabla_{h} \underline{w}^{\varepsilon, 3}-\Delta_{h} \underline{w}^{\varepsilon, 3}-\varepsilon^{2} \partial_{3}^{2} \underline{w}^{\varepsilon, 3}\right)\left(t, x_{h}, \varepsilon x_{3}\right)+\varepsilon \widetilde{F}^{\varepsilon}\left(t, x_{h}, \varepsilon x_{3}\right)
\end{aligned}
$$

where

$$
\widetilde{F}^{\varepsilon}\left(t, x_{h}, y_{3}\right) \stackrel{\text { def }}{=}\left(\left(\varepsilon \underline{w}^{\varepsilon} \cdot \nabla \underline{w}^{\varepsilon, h}, \underline{w}^{\varepsilon} \cdot \nabla \underline{w}^{\varepsilon, 3}\right)+\left(\underline{w}^{\varepsilon} \cdot \nabla \underline{v}^{h}, 0\right)+\varepsilon\left(\partial_{3}^{2} \underline{v}^{h}, 0\right)\right)\left(t, x_{h}, y_{3}\right) .
$$


In order to simplify the notation, let us write $\widetilde{F}^{\varepsilon}=\widetilde{F}^{\varepsilon, 1}+\widetilde{F}^{\varepsilon, 2}$ with

$$
\begin{aligned}
& \widetilde{F}^{\varepsilon, 1} \stackrel{\text { def }}{=}\left(\varepsilon \underline{w}^{\varepsilon} \cdot \nabla \underline{w}^{\varepsilon, h}, \underline{w}^{\varepsilon} \cdot \nabla \underline{w}^{\varepsilon, 3}\right)+\left(\underline{w}^{\varepsilon} \cdot \nabla \underline{v}^{h}, 0\right) \quad \text { and } \\
& \tilde{F}^{\varepsilon, 2} \stackrel{\text { def }}{=} \varepsilon\left(\partial_{3}^{2} \underline{v}^{h}, 0\right) \text {. }
\end{aligned}
$$

Recalling the equations satisfied by $\underline{v}^{h}$ and $\underline{w}^{\varepsilon}$, we infer that

$$
\left(\partial_{t} v_{a p p}^{\varepsilon}+v_{a p p}^{\varepsilon} \cdot \nabla v_{a p p}^{\varepsilon}-\Delta v_{a p p}^{\varepsilon}\right)\left(t, x_{h}, x_{3}\right)=-\nabla p_{a p p}^{\varepsilon}+\varepsilon G^{\varepsilon}\left(t, x_{h}, \varepsilon x_{3}\right)
$$

with $G^{\varepsilon}\left(t, x_{h}, y_{3}\right) \stackrel{\text { def }}{=}\left(\widetilde{F}^{\varepsilon}+\left(0, \partial_{3} \underline{p}_{0}\right)\right)\left(t, x_{h}, y_{3}\right), F^{\varepsilon}\left(t, x_{h}, x_{3}\right) \stackrel{\text { def }}{=} \varepsilon G^{\varepsilon}\left(t, x_{h}, \varepsilon x_{3}\right)$. Writing $q^{\varepsilon}=p^{\varepsilon}-p_{a p p}^{\varepsilon}$, we infer that

$$
\partial_{t} R^{\varepsilon}+R^{\varepsilon} \cdot \nabla R^{\varepsilon}+v_{\text {app }}^{\varepsilon} \cdot \nabla R^{\varepsilon}+R^{\varepsilon} \cdot \nabla v_{\text {app }}^{\varepsilon}-\Delta R^{\varepsilon}=-\nabla q^{\varepsilon}+F^{\varepsilon} .
$$

So Lemma 2.2 will be established as soon as we prove that $\left\|F^{\varepsilon}\right\|_{L^{2}\left(\mathbb{R}^{+} ; \dot{H}^{-\frac{1}{2}}\left(\mathbb{R}^{3}\right)\right)} \leq$ $C_{v_{0}, w_{0}} \varepsilon^{\frac{1}{3}}$.

The forcing term $F^{\varepsilon}$ consists of three different types of terms: a pressure term involving $\underline{p}_{0}$, a linear term $\varepsilon^{2} \partial_{3}^{2} \underline{v}^{h}\left(t, x_{h}, \varepsilon x_{3}\right)$, and finally a number of nonlinear terms, defined as $\varepsilon \widetilde{F}^{\varepsilon, 1}\left(t, x_{h}, \varepsilon x_{3}\right)$ above. Each of these contributions will be dealt with separately. Let us start with the pressure term.

Lemma 4.1. The following estimate holds:

$$
\varepsilon\left\|\left(\partial_{3} \underline{p}_{0}\right)\left(t, x_{h}, \varepsilon x_{3}\right)\right\|_{L^{2}\left(\mathbb{R}^{+} ; \dot{H}^{-\frac{1}{2}}\left(\mathbb{R}^{3}\right)\right)} \leq C_{v_{0}, w_{0}} \varepsilon^{\frac{1}{3}} .
$$

Proof. We define $P_{0}^{\varepsilon}\left(t, x_{h}, x_{3}\right) \stackrel{\text { def }}{=}\left(\partial_{3} \underline{p}_{0}\right)\left(t, x_{h}, \varepsilon x_{3}\right)$. Sobolev embeddings enable us to write

$$
\begin{aligned}
\left\|P_{0}^{\varepsilon}\right\|_{L^{2}\left(\mathbb{R}^{+} ; \dot{H}^{-\frac{1}{2}}\left(\mathbb{R}^{3}\right)\right)} & \lesssim\left\|P_{0}^{\varepsilon}\right\|_{L^{2}\left(\mathbb{R}^{+} ; L^{\frac{3}{2}}\left(\mathbb{R}^{3}\right)\right)} \\
& \lesssim \varepsilon^{-\frac{2}{3}}\left\|\partial_{3} \underline{p}_{0}\right\|_{L^{2}\left(\mathbb{R}^{+} ; L^{\frac{3}{2}}\left(\mathbb{R}^{3}\right)\right)}
\end{aligned}
$$

Recalling that

$$
\underline{p}_{0}=\left(-\Delta_{h}\right)^{-1} \sum_{j, k=1}^{2} \partial_{j} \partial_{k}\left(\underline{v}^{j} \underline{v}^{k}\right),
$$

we have by Sobolev embeddings that

$$
\begin{aligned}
\left\|\partial_{3} \underline{p}_{0}\right\|_{L^{2}\left(\mathbb{R}^{+} ; L^{\frac{3}{2}}\left(\mathbb{R}^{3}\right)\right)} & \lesssim \sum_{j, k=1}^{2}\left\|\underline{v}^{j} \partial_{3} \underline{v}^{k}\right\|_{L^{2}\left(\mathbb{R}^{+} ; L^{\frac{3}{2}}\left(\mathbb{R}^{3}\right)\right)} \\
& \lesssim\|\underline{v}\|_{L^{\infty}\left(\mathbb{R}^{+} ; L^{3}\left(\mathbb{R}^{3}\right)\right)}\left\|\partial_{3} \underline{v}\right\|_{L^{2}\left(\mathbb{R}^{+} ; L^{3}\left(\mathbb{R}^{3}\right)\right)} \\
& \lesssim\|\underline{v}\|_{L^{2}\left(\mathbb{R}^{+} ; H^{\frac{1}{2}}\left(\mathbb{R}^{3}\right)\right)}\left\|\partial_{3} \underline{v}\right\|_{L^{\infty}\left(\mathbb{R}^{+} ; H^{\frac{1}{2}}\left(\mathbb{R}^{3}\right)\right)},
\end{aligned}
$$

so we can conclude by using Lemma 3.1. This proves Lemma 4.1 .

Now let us consider the linear term $\varepsilon^{2} \partial_{3}^{2} \underline{v}^{h}\left(t, x_{h}, \varepsilon x_{3}\right)$. The statement is the following.

Lemma 4.2. The following estimate holds:

$$
\varepsilon^{2}\left\|\left(\partial_{3}^{2} \underline{v}^{h}\right)\left(t, x_{h}, \varepsilon x_{3}\right)\right\|_{L^{2}\left(\mathbb{R}^{+} ; \dot{H}^{-\frac{1}{2}}\left(\mathbb{R}^{3}\right)\right)} \leq C_{v_{0}} \varepsilon^{\frac{1}{2}} .
$$


Proof. We have

$$
\begin{aligned}
\varepsilon^{2}\left\|\left(\partial_{3}^{2} \underline{v}^{h}\right)\left(t, x_{h}, \varepsilon x_{3}\right)\right\|_{L^{2}\left(\mathbb{R}^{+} ; \dot{H}^{-\frac{1}{2}}\left(\mathbb{R}^{3}\right)\right)} & \lesssim \varepsilon\left\|\partial_{3}\left(\partial_{3} \underline{v}^{h}\left(t, x_{h}, \varepsilon x_{3}\right)\right)\right\|_{L^{2}\left(\mathbb{R}^{+} ; \dot{H}^{-\frac{1}{2}}\left(\mathbb{R}^{3}\right)\right)} \\
& \lesssim \varepsilon\left\|\left(\partial_{3} \underline{v}^{h}\right)\left(t, x_{h}, x_{3}\right)\right\|_{L^{2}\left(\mathbb{R}^{+} ; \dot{H}^{\frac{1}{2}}\left(\mathbb{R}^{3}\right)\right)} .
\end{aligned}
$$

A computation in Fourier variables shows that for any function $a$ on $\mathbb{R}^{3}$, we have

$$
\begin{aligned}
\left\|a\left(x_{h}, \varepsilon x_{3}\right)\right\|_{\dot{H}^{\frac{1}{2}\left(\mathbb{R}^{3}\right)}}^{2} & =\frac{1}{\varepsilon^{2}} \int_{\mathbb{R}^{3}}\left|\widehat{a}\left(\xi_{h}, \frac{\xi_{3}}{\varepsilon}\right)\right|^{2}|\xi| d \xi \\
& \leq \frac{1}{\varepsilon} \int_{\mathbb{R}^{3}}\left|\widehat{a}\left(\xi_{h}, \frac{\xi_{3}}{\varepsilon}\right)\right|^{2}\left|\xi_{h}\right| d \xi_{h} \frac{d \xi_{3}}{\varepsilon} \\
& +\int_{\mathbb{R}^{3}}\left|\widehat{a}\left(\xi_{h}, \frac{\xi_{3}}{\varepsilon}\right)\right|^{2} \frac{\left|\xi_{3}\right|}{\varepsilon} d \xi_{h} \frac{d \xi_{3}}{\varepsilon}
\end{aligned}
$$

By interpolation, we deduce that

$$
\left\|a\left(x_{h}, \varepsilon x_{3}\right)\right\|_{\dot{H}^{\frac{1}{2}\left(\mathbb{R}^{3}\right)}}^{2} \leq \frac{1}{\varepsilon}\|a\|_{L^{2}\left(\mathbb{R}^{3}\right)}\left\|\nabla_{h} a\right\|_{L^{2}\left(\mathbb{R}^{3}\right)}+\|a\|_{L^{2}\left(\mathbb{R}^{3}\right)}\left\|\partial_{3} a\right\|_{L^{2}\left(\mathbb{R}^{3}\right)} .
$$

Applying this inequality with $a=\partial_{3} \underline{v}$, we get

$$
\begin{aligned}
& \varepsilon^{2}\left\|\left(\partial_{3}^{2} \underline{v}^{h}\right)\left(t, x_{h}, \varepsilon x_{3}\right)\right\|_{L^{2}\left(\mathbb{R}^{+} ; \dot{H}^{-\frac{1}{2}}\left(\mathbb{R}^{3}\right)\right)} \\
& \vdots \varepsilon^{\frac{1}{2}}\left\|\partial_{3} \underline{v}(t)\right\|_{L^{2}\left(\mathbb{R}^{+} ; L^{2}\left(\mathbb{R}^{3}\right)\right)}^{\frac{1}{2}}\left\|\partial_{3} \nabla_{h} \underline{v}(t)\right\|_{L^{2}\left(\mathbb{R}^{+} ; L^{2}\left(\mathbb{R}^{3}\right)\right)}^{\frac{1}{2}} \\
& \quad+\varepsilon\left\|\partial_{3} \underline{v}(t)\right\|_{L^{2}\left(\mathbb{R}^{+} ; L^{2}\left(\mathbb{R}^{3}\right)\right)}^{\frac{1}{2}}\left\|\partial_{3}^{2} \underline{v}(t)\right\|_{L^{2}\left(\mathbb{R}^{+} ; L^{2}\left(\mathbb{R}^{3}\right)\right)}^{\frac{1}{2}} \\
& \leq C_{v_{0}} \varepsilon^{\frac{1}{2}}
\end{aligned}
$$

by Lemma 3.1, This proves Lemma 4.2 .

Now let us turn to the nonlinear terms that make up $F^{\varepsilon}$, which we denoted above by $\varepsilon \widetilde{F}^{\varepsilon, 1}$.

Lemma 4.3. The following estimate holds:

$$
\varepsilon\left\|\widetilde{F}^{\varepsilon, 1}\left(t, x_{h}, \varepsilon x_{3}\right)\right\|_{L^{2}\left(\mathbb{R}^{+} ; \dot{H}^{-\frac{1}{2}}\left(\mathbb{R}^{3}\right)\right)} \leq C_{v_{0}, w_{0}} \varepsilon^{\frac{1}{3}} .
$$

Proof. We recall that

$$
\widetilde{F}^{\varepsilon, 1}=\left(\varepsilon \underline{w}^{\varepsilon} \cdot \nabla \underline{w}^{\varepsilon, h}, \underline{w}^{\varepsilon} \cdot \nabla \underline{w}^{\varepsilon, 3}\right)+\left(\underline{w}^{\varepsilon} \cdot \nabla \underline{v}^{h}, 0\right) .
$$

Notice that for all functions $a$ and $b$ and any $1 \leq j \leq 3$,

$$
\begin{aligned}
\left\|a \partial_{j} b\right\|_{L^{2}\left(\mathbb{R}^{+} ; \dot{H}^{-\frac{1}{2}}\left(\mathbb{R}^{3}\right)\right)} & \lesssim\left\|a \partial_{j} b\right\|_{L^{2}\left(\mathbb{R}^{+} ; L^{\frac{3}{2}}\left(\mathbb{R}^{3}\right)\right)} \\
& \lesssim\|a\|_{L^{\infty}\left(\mathbb{R}^{+} ; L^{3}\left(\mathbb{R}^{3}\right)\right)}\left\|\partial_{j} b\right\|_{L^{2}\left(\mathbb{R}^{+} ; L^{3}\left(\mathbb{R}^{3}\right)\right)} .
\end{aligned}
$$

Defining $c^{\varepsilon}\left(t, x_{h}, x_{3}\right)=\left(a \partial_{j} b\right)\left(t, x_{h}, \varepsilon x_{3}\right)$, this implies that

$$
\left\|c^{\varepsilon}\right\|_{L^{2}\left(\mathbb{R}^{+} ; \dot{H}^{-\frac{1}{2}}\left(\mathbb{R}^{3}\right)\right)} \lesssim \varepsilon^{-\frac{2}{3}}\|a\|_{L^{\infty}\left(\mathbb{R}^{+} ; \dot{H}^{\left.\frac{1}{2}\left(\mathbb{R}^{3}\right)\right)}\right.}\left\|\partial_{j} b\right\|_{L^{2}\left(\mathbb{R}^{+} ; \dot{H}^{\frac{1}{2}}\left(\mathbb{R}^{3}\right)\right)} .
$$

We can apply this inequality with $a$ and $b$ equal to $\underline{v}$ or $\underline{w}^{\varepsilon}$, due to the results proved in Section 3, and the lemma follows. 


\section{REFERENCES}

[1] M. Cannone, Y. Meyer and F. Planchon, Solutions autosimilaires des équations de NavierStokes, Séminaire "Équations aux Dérivées Partielles" de l'École polytechnique, Exposé VIII, 1993-1994. MR 1300903 (95k:35157)

[2] J.-Y. Chemin, Remarques sur l'existence pour le système de Navier-Stokes incompressible, SIAM Journal on Mathematical Analysis, 23, 1992, pages 20-28. MR1145160 (93a:35118)

[3] J.-Y. Chemin and I. Gallagher, On the global wellposedness of the 3-D Navier-Stokes equations with large initial data, Annales de l'École Normale Supérieure, 39, 2006, pages 679-698. MR2290141

[4] J.-Y. Chemin and I. Gallagher, Wellposedness and stability results for the Navier-Stokes equations in $\mathbf{R}^{3}$, Annales de l'Institut Henri Poincaré, Analyse Non Linéaire, 26, 2009, pages 599-624. MR2504045

[5] H. Fujita and T. Kato, On the Navier-Stokes initial value problem I, Archive for Rational Mechanics and Analysis, 16, 1964, pages 269-315. MR0166499(29:3774)

[6] I. Gallagher, The tridimensional Navier-Stokes equations with almost bidimensional data: stability, uniqueness and life span, International Mathematical Research Notices, 18, 1997, pages 919-935. MR:1481611 (98m:76049)

[7] I. Gallagher, D. Iftimie and F. Planchon, Asymptotics and stability for global solutions to the Navier-Stokes equations, Annales de l'Institut Fourier, 53, 2003, no. 5, pages 1387-1424. MR:2032938 (2005b:35219)

[8] Y. Giga and T. Miyakawa, Solutions in $L^{r}$ of the Navier-Stokes initial value problem, Archive for Rational Mechanics and Analysis, 89, 1985, no. 3, pages 267-281. MR786550|(86m:35138)

[9] D. Iftimie, The 3D Navier-Stokes equations seen as a perturbation of the 2D NavierStokes equations, Bulletin de la Société Mathématique de France, 127, 1999, pages 473-517. MR:1765551(2001e:35139)

[10] D. Iftimie, G. Raugel and G.R. Sell, Navier-Stokes equations in thin 3D domains with Navier boundary conditions, Indiana University Mathematics Journal, 56, 2007, pages 1083-1156. MR2333468

[11] H. Koch and D. Tataru, Well-posedness for the Navier-Stokes equations, Advances in Mathematics, 157, 2001, pages 22-35. MR.1808843(2001m:35257)

[12] O. Ladyzhenskaya, The mathematical theory of viscous incompressible flow. Second English edition, revised and enlarged. Mathematics and its Applications, Vol. 2. Gordon and Breach Science Publishers, New York-London-Paris, 1969, xviii+224 pp. MR0254401 (40:7610)

[13] S. Leibovich, A. Mahalov and E. Titi, Invariant helical subspaces for the Navier-Stokes equations. Arch. Rational Mech. Anal., 112, 1990, no. 3, pages 193-222. MR1076072(91h:35252)

[14] J. Leray, Essai sur le mouvement d'un liquide visqueux emplissant l'espace, Acta Matematica, 63, 1933, pages 193-248.

[15] A. Mahalov and B. Nicolaenko, Global solvability of three-dimensional Navier-Stokes equations with uniformly high initial vorticity, (Russian. Russian summary) Uspekhi Mat. Nauk, 58, 2003, pages 79-110; translation in Russian Math. Surveys, 58, 2003, pages 287-318. MR.1992565 (2004h:35177)

[16] G. Ponce, R. Racke, T. Sideris and E. Titi, Global stability of large solutions to the 3D Navier-Stokes equations, Comm. Math. Phys., 159, 1994, no. 2, pages 329-341. MR.1256992 (95a:35115)

[17] G. Raugel and G.R. Sell, Navier-Stokes equations on thin 3D domains. I. Global attractors and global regularity of solutions, Journal of the American Mathematical Society, 6, 1993, pages 503-568. MR.1179539 (93j:35134)

[18] M. Ukhovskii and V. Iudovich, Axially symmetric flows of ideal and viscous fluids filling the whole space, Prikl. Mat. Meh., 32, 1968, pages 59-69 (Russian); translated as J. Appl. Math. Mech., 32, 1968, pages 52-61. MR0239293(39:650)

Laboratoire J.-L. Lions UMR 7598, Université Paris VI, 175 RUe du Chevaleret, 75013 PARIS, France

E-mail address: chemin@ann.jussieu.fr

Institut de Mathématiques de Jussieu UMR 7586, Université Paris VII, 175 RUe DU Chevaleret, 75013 Paris, France

E-mail address: Isabelle.Gallagher@math.jussieu.fr 